\title{
SITUATION DES POISSONS MIGRATEURS AMPHIHALINS SUR LE BASSIN DE L'ADOUR.
}

\section{A. MARTY et B. BOUSQUET}

Conseil Supérieur de la Pêche, Délégation Régionale Midi-Pyrénées Aquitaine,

Building des Pyrénées 2D, 64000 Pau, France.

\section{RÉSUMÉ}

L'Adour (drainant $17000 \mathrm{~km}^{2}$ sur la façade atlantique, exploités par la pêche amateur et la pêche professionnelle) est fréquenté par six grands migrateurs amphihalins: saumon atlantique, truite de mer, anguille, grande alose, alose feinte et lamproie marine, tous plus ou moins en voie de régression au début des années 1980. Au cours des deux dernières décennies, des actions diverses (aménagements du milieu, études, repeuplements, mesures réglementaires) ont été menées pour leur restauration. Ces actions ont pu être conduites grâce à des concours financiers (Etat, Région, Agence de l'Eau, Europe) et à la participation des pêcheurs regroupés au sein de l'association MIGRADOUR, avec l'appui technique du Conseil Supérieur de la Pêche. La présente communication fait le point sur chacune de ces espèces, conformément à un plan d'exposé en trois parties : état initial (avant 1980), actions menées de 1980 à 1998 et bilan et, orientation actuelle.

\section{SITUATION OF AMPHYALINE MIGRATORY FISHES IN ADOUR RIVER.}

\section{ABSTRACT}

The Adour River, on the Atlantic coast in SW France with a watershed of $17,000 \mathrm{~km}^{2}$, holds populations of 6 amphihaline migratory species (Atlantic salmon, sea trout, eel, two shads and sea lamprey) exploited by commercial and sport fisheries. In the early ' 80 s, all these populations were more or less declining. Various actions (habitat improvement, population studies, stocking, fishing regulations) were undertaken during the last two decades for their enhancement, with the financial support of regional, state and European administrations and regional water agency, and with the participation of fishermen gathered within MIGRADOUR, supported technically by the CSP. This communication presents the status of the different species according to the following plan: status before 1980, actions undertaken between 1980 and 1998 and evaluation of results, current orientation. 


\section{INTRODUCTION}

Le bassin de l'Adour (Figure 1), d'une superficie de $17000 \mathrm{~km}^{2}$, s'étend sur quatre départements: Pyrénées-Atlantiques, Landes, Gers et Hautes-Pyrénées. II débouche dans l'Océan Atlantique. Les différents cours d'eau qui le composent ont des régimes hydrauliques de type océanique (fluvial) avec des étiages sévères pour l'axe Adour et les cours d'eau situés au nord du bassin, de type pluvio-nival à débits soutenus pour les gaves.

Historiquement, le bassin de l'Adour a toujours accueilli des migrateurs amphihalins. Actuellement, six espèces sont présentes: le saumon, la truite de mer, la grande alose, l'alose feinte, l'anguille et la lamproie marine. L'esturgeon, qui fréquentait l'Adour jusque dans la première moitié du XX ${ }^{\text {eme }}$ siècle, a aujourd'hui disparu.

Depuis les années 1970, de nombreux pêcheurs (professionnels et à la ligne) s'inquiétaient de la diminution de leurs captures de "grands migrateurs ". Alerté, le Ministère de l'Environnement a lancé en 1976 le premier plan "saumon", incluant des programmes d'études et actions visant à évaluer l'état des stocks, apprécier les causes éventuelles de leur diminution et engager des programmes de restauration. En parallèle, un travail législatif et réglementaire était mené au plan national : modification de la loi de 1919 sur l'hydroélectricité en 1980, nouvelle loi pêche en 1984, loi sur l'eau en 1992, décret " amphihalins" de 1994 instituant les comités de gestion des poissons migrateurs.

Le présent article se propose:

- en premier lieu, de présenter globalement le travail mené sur l'Adour en faveur des grands migrateurs amphihalins; espèce.

- et, en second lieu, de rentrer plus dans le détail de ces actions, espèce par

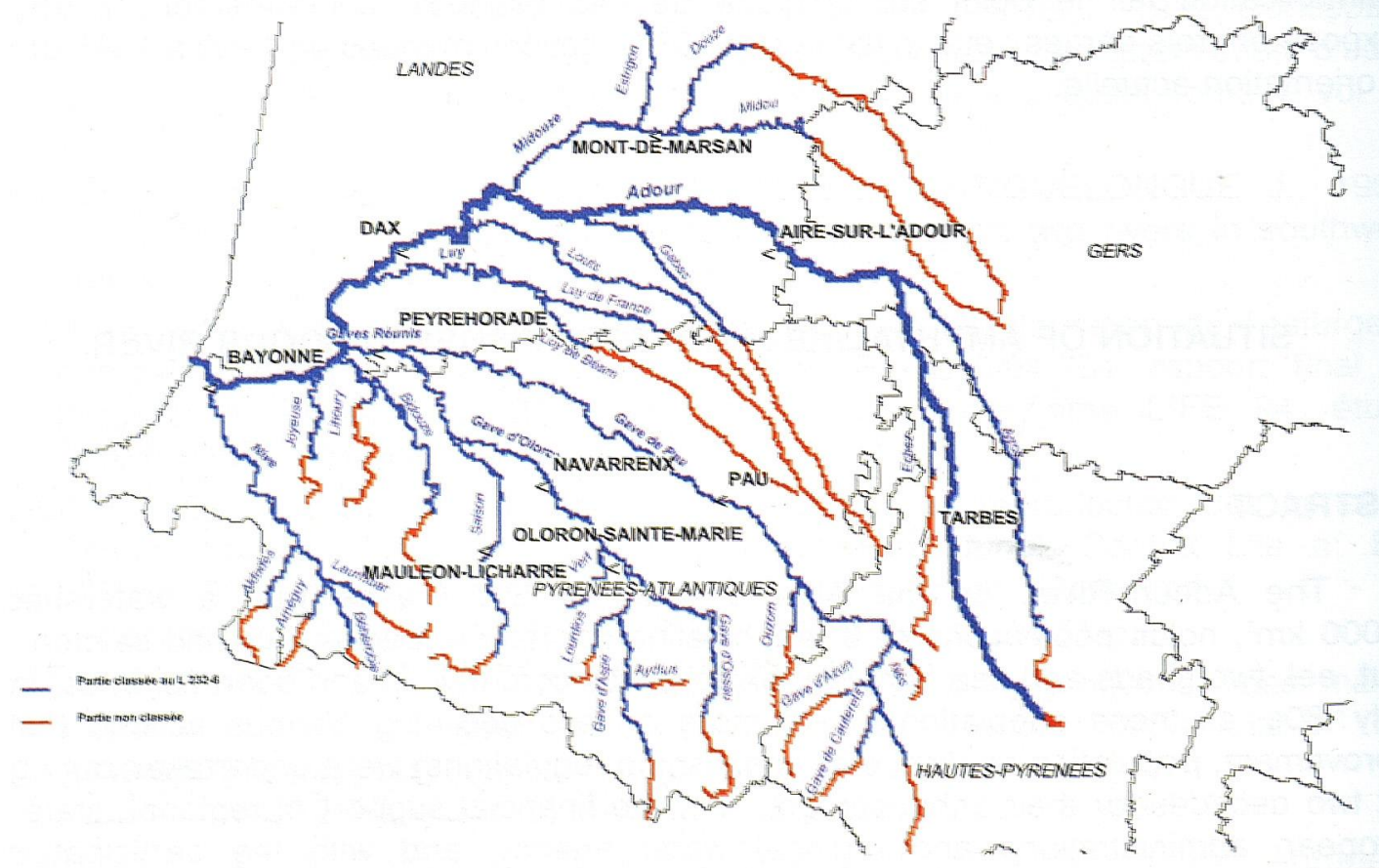

Figure 1

Bassin de l'Adour.

Figure 1

The Adour River. 


\section{BILAN GÉNÉRAL DES ACTIONS MENÉES SUR L'ADOUR EN FAVEUR DES AMPHIHALINS}

Sur la période allant du lancement du premier «Plan saumon » (1976) à 1999, les différentes actions engagées sur l'Adour à l'attention des amphihalins se marquent globalement par:

- le classement de trente trois cours d'eau au titre de l'article $L 232.6$ du Code rural (cours d'eau soumis à dispositifs de franchissement), soit un linéaire total de $1500 \mathrm{~km}$ (Figure 1);

- la réalisation de 140 dispositifs de franchissement (Tableau I) ;

\section{Tableau I}

Etat d'avancement du programme d'équipement des barrages du bassin de

l'Adour en dispositifs de franchissement.

\section{Table I}

Actual situation of Adour River fishways program.

\begin{tabular}{|c|c|c|c|c|c|}
\hline $\begin{array}{c}\text { Cours d'eau } \\
\text { et/ou } \\
\text { sous bassin }\end{array}$ & \multicolumn{2}{|c|}{ de franchissements réalisés } & $\begin{array}{c}\text { Km ouverts } \\
\text { ou } \\
\text { améliorés }\end{array}$ & $\begin{array}{c}\text { Dispositifs } \\
\text { de franchissement restant } \\
\text { à faire ou améliorer }\end{array}$ \\
\cline { 2 - 6 } & $\begin{array}{c}\text { passes à } \\
\text { poissons }\end{array}$ & dévalaison & \multicolumn{2}{|c|}{$\begin{array}{c}\text { passes à } \\
\text { poissons }\end{array}$} & dévalaison \\
\hline Adour & 11 & 2 & 170 & 28 & 9 \\
\hline $\begin{array}{c}\text { Midouze, Luy, } \\
\text { Bidouze, Lihoury, } \\
\text { Aran }\end{array}$ & 4 & 0 & 153 & 38 & 6 \\
\hline $\begin{array}{c}\text { Gave de Pau } \\
\text { et affl. }\end{array}$ & 29 & 10 & 159 & 9 & 11 \\
\hline $\begin{array}{c}\text { Gave d'Oloron } \\
\text { et affl. }\end{array}$ & 34 & 23 & 225 & 8 & 14 \\
\hline $\begin{array}{c}\text { Nive } \\
\text { et affl. }\end{array}$ & 20 & 7 & 92 & 2 & 6 \\
\hline $\begin{array}{c}\text { Total Bassin } \\
\text { Adour }\end{array}$ & 98 & 42 & 799 & 85 & 46 \\
\hline
\end{tabular}

- la création de six stations de contrôle des migrateurs ;

- la mise en place de quatre structures de production de juvéniles de saumon atlantique et de truite de mer;

- la prise de mesures réglementaires de gestion pour le saumon et la civelle (cible d'échappement et quotas de captures pour le saumon, restriction des périodes de pêche pour les pêcheurs amateurs de civelle);

- le soutien des effectifs à travers des mesures de repeuplements (saumon) et de transfert de populations (anguille);

- la prise de mesures de protection pour le milieu aquatique (arrêts d'extractions de granulats, arrêtés de biotopes) ;

- la réalisation d'études visant à développer la connaissance des stocks, la biologie de certaines espèces et des outils de gestion ;

- la mise en cuvre de quelques actions ponctuelles de remise en état du milieu. 
Une grande part de ces actions n'a pu être menée à bien que grâce à la participation de plusieurs partenaires financiers dont les principaux sont l'Etat, les Régions Aquitaine et Midi-Pyrénées (volet "Restauration des migrateurs » dans les contrats de plan Etat-Régions Aquitaine et Midi-Pyrénées), l'Agence de l'Eau Adour Garonne, l'Europe, le Conseil Supérieur de la Pêche et les collectivités piscicoles.

L'engagement des pêcheurs s'est exprimé depuis 1995 par la création d'une association, MIGRADOUR, regroupant les pêcheurs à la ligne et professionnels fluviaux.

\section{SITUATION PAR ESPÈCE}

\section{Saumon atlantique}

Autrefois abondants (plus de 10000 captures annuelles à la tin du siècle dernier), les saumons atlantiques du bassin de l'Adour n'ont cessé de se raréfier au cours du $X X^{\text {ème }}$ siècle, tombant à moins de 500 captures par an au milieu des années 1970 (BOUSQUET et MARTY, 1987). Devant ce déclin, un plan de restauration, initié par le Ministère de l'Environnement, a été lancé en 1976. Ce plan s'appuyait sur l'existence d'un énorme potentiel de production de juvéniles non utilisé du fait de l'édification ou du rehaussement de nombreux barrages au fil des années, interdisant l'accès des géniteurs aux parties hautes du bassin. Un chiffrage a fait apparaître que la réouverture complète des axes Gaves et Nive ferait passer le potentiel, alors estimé entre 50 et 100000 smolts, à plus de 400000 smolts.

Des passes à poissons ont donc été construites et l'objectif de libre circulation sur les axes Gaves et Nive est aujourd'hui atteint à plus de $80 \%$ (83 dispositifs de franchissement réalisés à ce jour). Seul le gave de Pau pose encore de sérieux problèmes, le fonctionnement de plusieurs passes anciennes devant être amélioré (CHANSEAU, 1998).

Dès le démarrage du plan, une politique d'alevinage a par ailleurs été menée pour renforcer les effectifs et accélérer le processus de relance du stock (notamment, la construction, puis la mise en production en 1983 de l'écloserie de Préchacq, sur le gave d'Oloron, d'une capacité d'accueil de 300000 alevins). S'agissant de l'exploitation, coexistaient en 1976 - et coexistent encore aujourd'hui - trois pêcheries : (1) à l'aval, deux pêcheries professionnelles aux engins, la première soumise à la réglementation maritime (en moyenne, $80 \%$ des prises de saumon), la seconde relevant de la réglementation fluviale (10\% des prises); (2) à l'amont, une pêcherie à la ligne, soumise à la réglementation fluviale (10\% des prises). Le souci de mieux protéger les géniteurs a amené le Ministère de l'Environnement à renforcer la réglementation fluviale. C'est ainsi que, notamment, des quotas de captures ont été imposés à partir de 1987 aux pêcheurs fluviaux (lignes et engins). Le Ministère de la Mer s'est montré plus libéral en n'exigeant pas de quotas de ses pêcheurs aux engins.

Le suivi de plusieurs indicateurs de l'état de la population de saumon (captures aux engins et à la ligne, stations de piégeage, frayères, recrutement naturel et artificiel) a été mis en place, au cours de la période couverte par le plan. Ces collectes de données, menées de concert par différents intervenants (Conseil Supérieur de la Pêche, MIGRADOUR, Institut Français de Recherche pour l'Exploitation de la MER, associations de pêche...) permettent aujourd'hui d'avoir une vision croisée de l'évolution du stock.

Ainsi constate-t-on que, sur la période 1980-1995 (prise comme période de référence dans les lignes qui suivent): 
- la tendance à la baisse des effectifs a été stoppée mais l'espoir d'un renversement de situation ne s'est pas concrétisé (Figure 2) ;

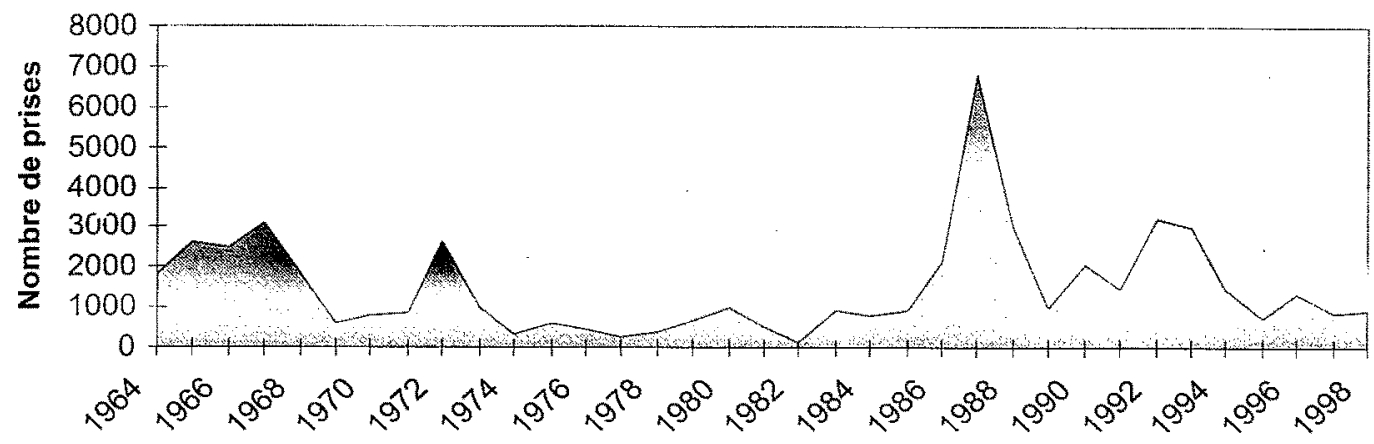

Années de capture

\section{Figure 2}

\section{Variations du nombre de saumons atlantiques capturés sur l'Adour, tous moyens de pêche confondus, de 1964 à 1998.}

\section{Figure 2}

\section{Variation of number of Atlantic salmon caught in Adour River (net + rod) from 1964 to 1998.}

- des analyses statistiques (tests de corrélation des rangs de Spearman) font apparaître que le soutien des stocks par le biais des alevinages n'a pas eu, jusqu'à présent, les bénéfices escomptés : certes, les alevinages ont généré des retours d'adultes significatifs (seuil $\alpha=0,01$ ), mais ces adultes étaient principalement des poissons d'1 an de mer qui n'ont pas (ou ont peu) participé au renforcement du recrutement naturel: corrélation significative entre recrutements naturels et retours d'adultes 2 ans de mer (seuil $\alpha=0,1$ ), non significative entre recrutements naturels et retours de 1 an de mer (seuil $\alpha=0,1$ );

- alors que le programme d'ouverture des barrages infranchissables a multiplié par deux les surfaces de production des juvéniles théoriquement accessibles, la recolonisation des parties hautes du réseau ne s'est pratiquement pas produite.

La réussite du plan étant sous-tendue à une progression régulière du nombre de géniteurs, l'absence de prise de mesures véritablement contraignantes, au plan de l'exploitation, revenait, de fait, à supposer que l'échappement irait « de lui-même » en croissant, au fur et à mesure de l'augmentation du volume des retours. Force est d'admettre aujourd'hui qu'un tel scénario ne s'est pas réalisé: les saumons ont manifestement été beaucoup plus exploités - y compris en valeurs relatives - les années où ils sont revenus nombreux que celles où ils étaient en faible nombre. Le résultat est que l'échappement a stagné à un niveau trop bas pour que la recolonisation des têtes de bassin se fasse correctement.

Une profonde révision des modalités de pêche, pour toutes les catégories de pêcheurs mais notamment, bien sûr, pour la pêche aux engins, s'imposait donc. Sous l'égide du COGEPOMI Adour, mis en place en 1996, une réflexion en profondeur a été menée entre les différents partenaires (CSP, IFREMER, Institut National de la Recherche Agronomique, Catégories de pêcheurs...) visant, d'abord, à déterminer une cible d'échappement en rapport avec le potentiel d'accueil, ensuite, à prendre les mesures réglementaires permettant d'atteindre cette cible. L'accord s'est négocié, dans un premier 
temps, sur une cible de 11 millions d'œufs (2 400 " équivalents géniteurs " Adour) et les dispositions suivantes ont notamment été adoptées, pour trois ans à partir de 1999 (ANONYME, 1998) : ligne ;

- ouverture retardée au 10 avril et quota global de 180 saumons pour la pêche à la

- 1 mois $1 / 2$ d'arrêt supplémentaire de la pêche aux engins dans la période juin - juillet.

Il va sans dire que le suivi des indicateurs déjà mis en place sera poursuivi - voire intensifié - afin de mesurer l'effet de ces nouvelles dispositions sur le stock.

\section{Truite de mer}

A la différence du saumon, étudié de longue date sur l'Adour (CHIMITS, 1936 ; VIBERT, 1950), la truite de mer n'avait fait l'objet, jusqu'à une période récente, d'aucun travail spécifique. Un tel manque d'intérêt trouve peut être une explication dans le fait que n'existe pas, sur le bassin, de tradition de pêche à la ligne de ce poisson. Quoi qu'il en soit, labsence de donnée rend impossible l'établissement de la situation historique du stock.

Depuis la mise en route des plans migrateurs, aucune action de préservation ou de restauration n'a véritablement été ciblée sur la truite de mer. Toutefois, cette espèce ayant une biologie voisine de celle du saumon, il a toujours été considéré que l'effort mené en faveur de ce dernier, notamment en matière de franchissement de barrages et d'accès aux frayères, profitait tout autant à la truite de mer.

S'agissant de la période actuelle, deux indicateurs principaux nous renseignent sur les populations de truites de mer de l'Adour: les captures annuelles déclarées par les pècheurs aux engins ef les contrôles menés au niveau des stations de piégeage.

- La Figure 3 fournit l'état des prises annuelles réalisées par les marins pêcheurs au cours de la période 1985-1997 (PROUZET et al, 1998). On note qu'après des captures de l'ordre de 800 à 1200 jusqu'en 1989, le débarquement s'est stabilisé depuis 9 ans entre 400 et 600 prises par an.

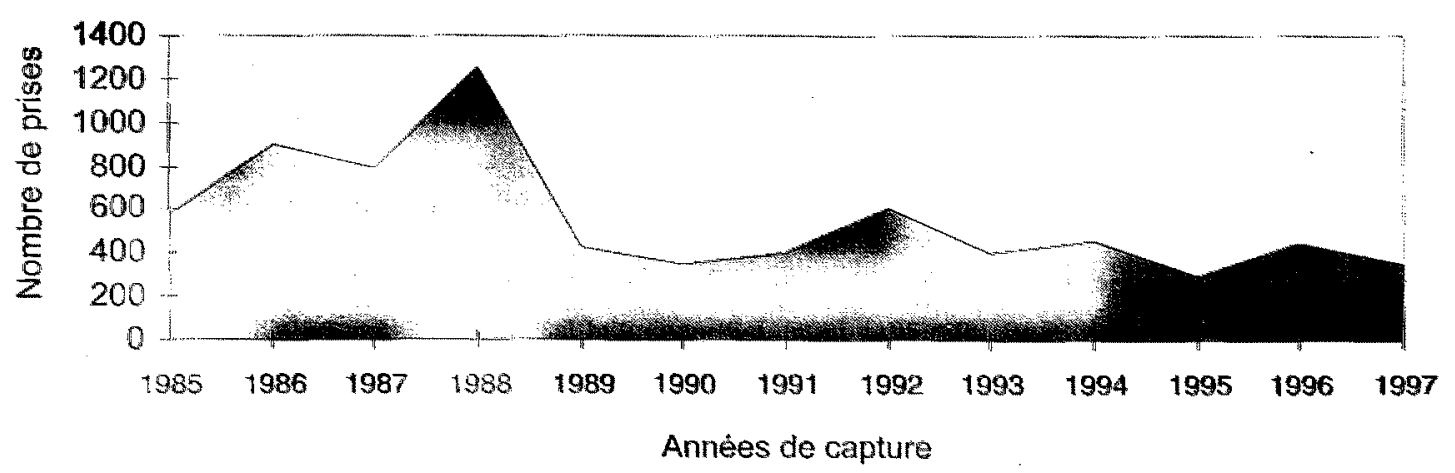

Figure 3

Variations du nombre de truites de mer capturées par les marins pêcheurs dans l'estuaire de l'Adour de 1985 à 1997. 
- mises en fonctionnement entre 1995 et 1997, les stations de piégeage ont permis de contrôler de 500 à 600 individus par an. Ces échantillonnages ont fourni des informations sur les rythmes migratoires, les caractéristiques biométriques et structures d'âges (DARROLLES, 1997) et ont permis une première évaluation des stocks rentrant dans le gave d'Oloron: 900 à 1800 individus (BARRACOU, 1999).

L'objectif, pour les années futures, est d'abord l'amélioration des connaissances sur cette espèce. Ainsi, parallèlement à la poursuite des observations au niveau des stations de cuntrôle, une étude de dynamique de population, incluant les deux écotypes eau douce et eau de mer, est d'ores et déjà programmée sur le gave de Mauléon.

S'agissant des prélèvements, les mesures conservatoires proposées par le Préfet sur proposition du COGEPOMI Adour pour mieux protéger les géniteurs de saumon devraient également être bénéfiques aux truites de mer.

\section{Anguille}

Elle constitue l'espèce la plus largement distribuée sur le bassin de l'Adour. La répartition des peuplements établie par le CSP à partir de 1700 inventaires ou sondages (Figure 4) fait apparaître une grande hétérogénéité, avec de forts niveaux d'abondance ( $>50$ individus estimés / $1000 \mathrm{~m}^{2}$ ) sur les zones les plus proches de l'océan $(<30 \mathrm{~km}$ ) et qui s'amenuisent vers l'amont.

On note l'absence d'anguilles sur les têtes de bassin des Gaves et de l'Adour et on remarque que globalement les populations sont en faible densité ou absentes sur plus de $70 \%$ du réseau hydrographique de l'Adour. En l'absence de données antérieures aux années 1970, il n'est pas possible de retracer l'évolution historique de la distribution de l'anguille en parallèle de l'aménagement des cours d'eau et de la dégradation des habitats aquatiques.

Toutefois, la cartographie des barrages construits depuis le début du XXme siècle ef les variations brusques de densités d'anguilles et/ou de structures de taille peuvent être mises en relation. Cette situation est marquée sur les gaves de Pau, Ossau, Aspe, Adour et traduit sans aucun doute l'impact important d'obstacles qui sont "étanches " aux migrations, ou ne deviennent que temporairement franchissables lors de crues.

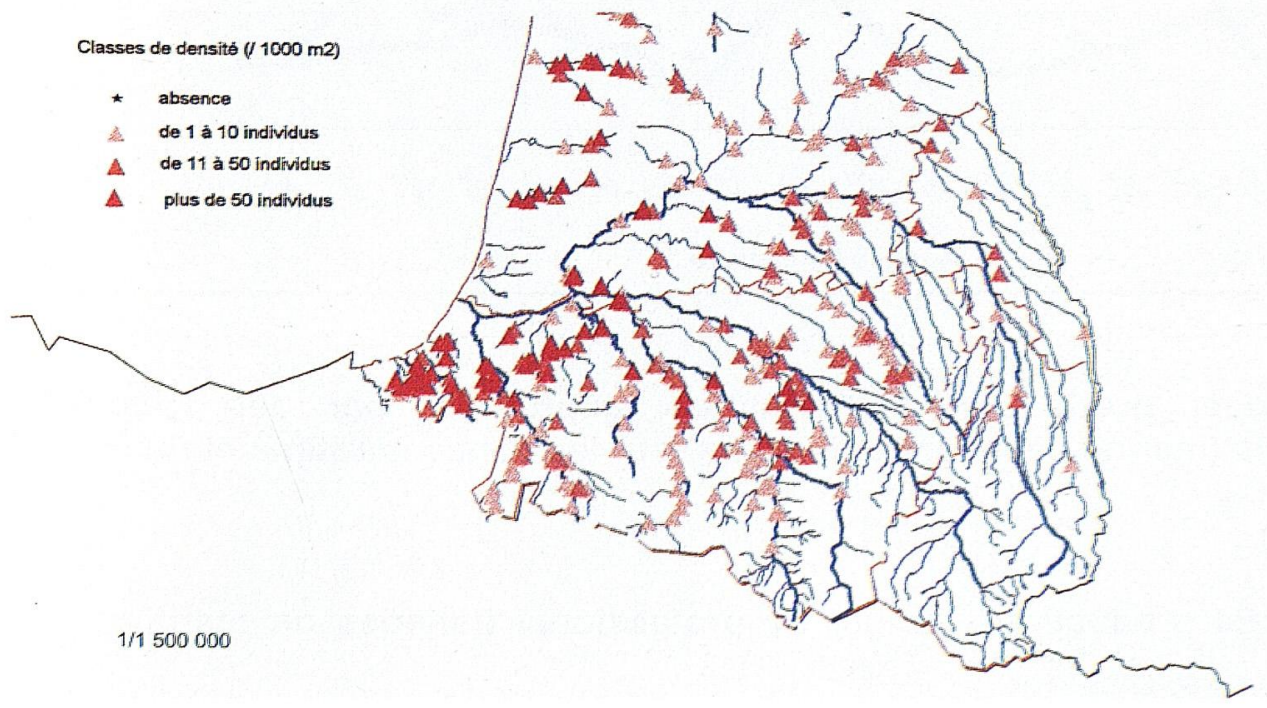

Figure 4

Distribution de l'anguille sur le bassin de l'Adour.

\section{Figure 4}

Eel distribution in Adour River. 
Il en est de même sur d'autres bassins où la transformation d'anciens ouvrages en usines hydroélectriques a fortement transformé les modalités de circulation des anguilles et diminué leurs peuplements.

Ces transformations ont réduit l'accessibilité aux zones de croissance potentielle et diminué ainsi substantiellement les capacités d'accueil du bassin Adour. Ces capacités sont par ailleurs altérées sur certains axes (Adour, Luy, Louts...) par une très forte aggravation des étiages naturels dus au développement intensif de l'irrigation ainsi que par l'altération de la qualité des eaux (rejets de la papeterie de la ville de Tartas).

Les populations d'anguilles pourraient également être influencées par l'état sanitaire des poissons. Une étude menée sur les 19 principaux cours d'eau de ce bassin en 1998 a montré que l'anguille hébergeait différents parasites (DANIEL et al., 1998). Parmi eux, le parasite Anguillicola crassus, apparu dans les années 1980, a ainsi été retrouvé sur l'ensemble des points d'études avec une prévalence moyenne de $55 \%$ (fourchette 50 à $70 \%$ ), sans toutefois qu'une progression géographique du taux de contamination ne puisse être mise en évidence entre 1994 et 1998 (DANIEL et al., 1998).

Concernant les prélèvements, jusqu'en 1995, la civelle était exclusivement pêchée à l'aide du tamis manié à la main. Depuis, la technique du drossage (tamis poussés) s'est développée en zone maritime, sans que l'on puisse évaluer les conséquences de cette nouvelle pêche sur les populations d'anguilles.

En ce qui concerne les déclarations de captures d'anguille, d'après PROUZET et al. (1998), les débarquements des marins pêcheurs exerçant sur l'estuaire (Figure 5) semblent traduire une baisse de l'abondance de l'espèce. Cette appréciation est toutefois difficile à confirmer à partir des déclarations des pêcheurs professionnels fluviaux faites à la Direction Départementale de l'Agriculture et des Forets des Landes (Figure 5).

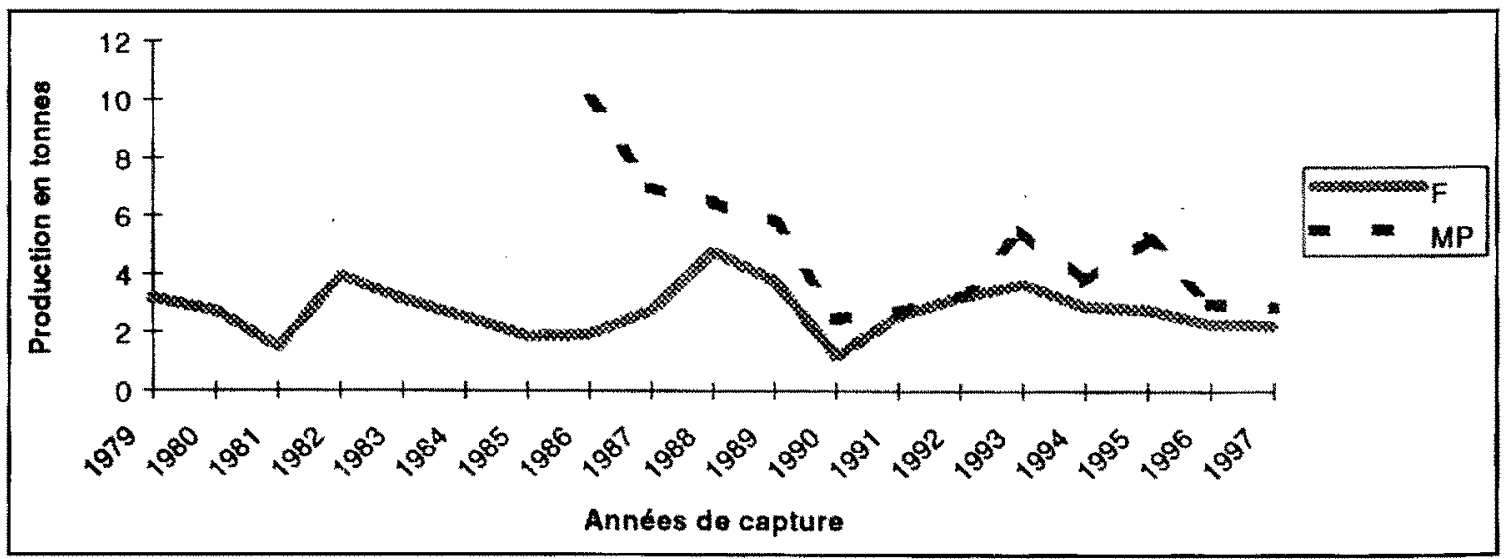

Figure 5

Variations des productions d'anguilles capturées par les pêcheurs professionnels (marins pêcheurs et fluviaux) sur les zones maritime et fluviale de l'Adour.

\section{Figure 5}

Variation of eel productions caught by professional fisheries on maritime and fluvial areas.

En revanche, et pour ce qui concerne le stade civelle, les captures des deux catégories de pêcheurs professionnels (Figure 6) indiquent une diminution des débarquements qui proviendrait d'un affaiblissement des stocks. 


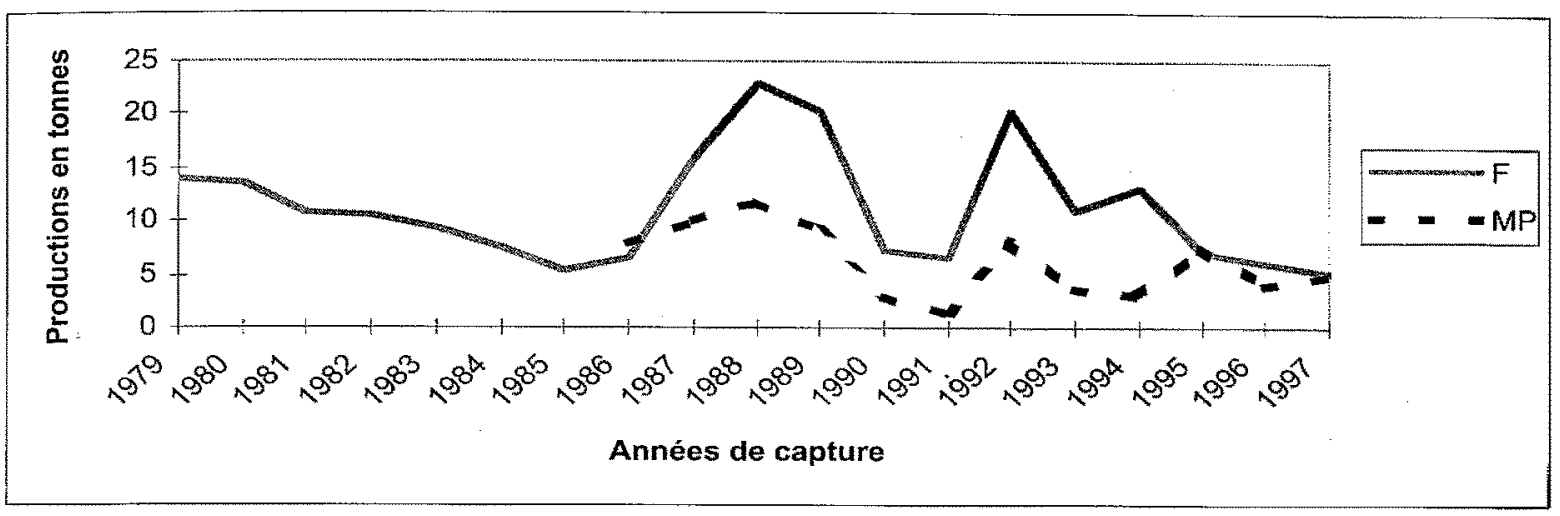

\section{Figure 6}

\section{Variations des productions de civelles capturées par les pêcheurs professionnels (marins pêcheurs ef fluviaux) sur les zones maritime et fluviale de l'Adour (déclaration).}

Figure 6

Variation of elver productions caught by professional fisheries on maritime and fluvial areas (declaration).

Depuis 1997, des outils de gestion visant à mieux appréhender l'évolution des populations sont en cours de mise en place. Il s'agit de l'évaluation des flux de civelles et de leur taux d'exploitation en zone estuarienne, ainsi que de la mise en place d'un réseau de stations de contrôle des anguilles par pêche électrique sur la zone fluviale.

Un programme de passes spécifiques à anguille et civelle est d'autre part en cours de réalisation pour rouvrir d'importantes zones de colonisation ne présentant pas de risque de mortalité à la dévalaison (lacs landais, Bidouze, Lihoury, Luy).

On notera enfin que des mesures réglementaires ont été prises en 1997 par le Préfet des Landes sur proposition du COGEPOMI Adour pour réduire l'effort de pêche des pêcheurs amateurs de civelles.

\section{Alose}

L'Adour est fréquenté par deux espèces d'aloses : la grande alose (Alosa alosa) et l'alose feinte (Alosa fallax). La cartographie de leurs aires de répartition montre qu'elles sont très influencées par les obstacles et l'altération des milieux aquatiques. Depuis la moitié du $X X^{\text {thm }}$ siècle, on a assisté à une réduction considérable des longueurs de cours d'eau colonisés (30\% sur le gave de Pau, $100 \%$ sur la Midouze, jusqu'à $70 \%$ sur l'Adour). Toutefois, la création d'une quinzaine de passes à poissons sur les cours d'eau à alose a permis de rétablir l'accès à de nombreuses frayères (BARRACOU, 1997).

Les aloses sont essentiellement capturées par les pêcheurs professionnels et amateurs aux engins au moyen respectivement de filets et carrelets. Une péche à la ligne s'exerce sur ces deux poissons sur l'aval du gave d'Oloron.

La Figure 7 retrace l'évolution des captures déclarées de grande alose par les marins pêcheurs au cours de la période 1986 - 1997 (PROUZET et al,, 1998). Les captures, de l'ordre de 20000 individus en 1986, ont régulièrement chuté pour atteindre un 
palier de 5000 prises dans la première moitié de la décennie 1990. Depuis, un redressement se manifeste. La même tendance est observée sur les captures des pêcheurs fluviaux.

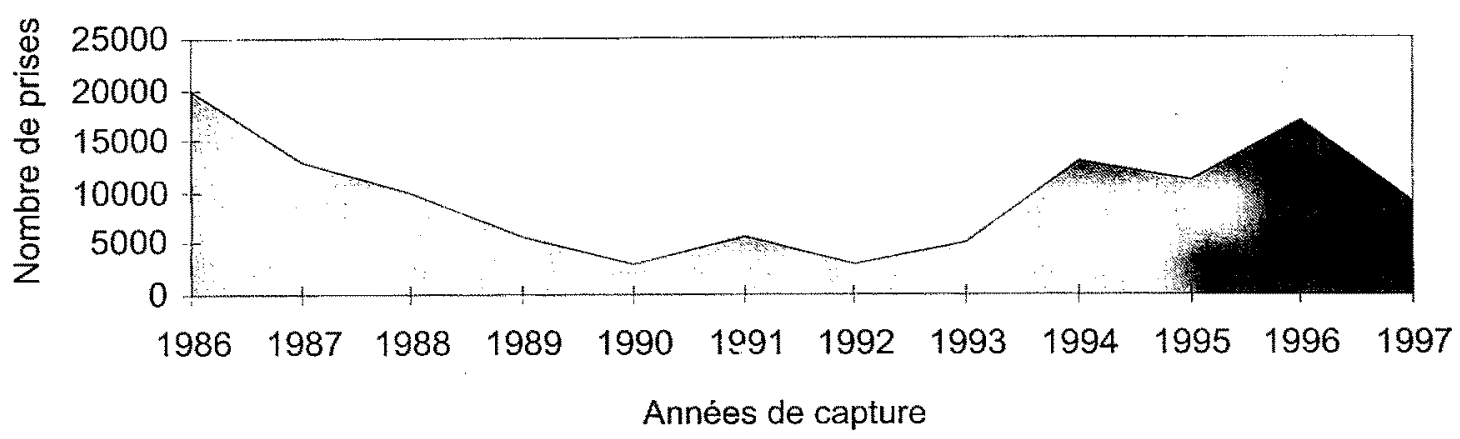

\section{Figure 7}

Variations du nombre de grandes aloses capturées par les marins pêcheurs dans l'estuaire de l'Adour de 1986 à 1997 (déclaration).

\section{Figure 7}

Variation of number of shad (Alosa alosa) caught by estuary net fishery from 1986 to 1997 (declaration).

La chute des effectifs est notamment attribuée à des problèmes d'accès aux zones de reproduction sur l'Adour (barrages d'Onard, St Sever) et à des étiages très sévères (1986) qui ont réduit de façon drastique les zones de grossissement des juvéniles. Ces problèmes sont en partie résolus aujourd'hui - à l'exception des débits d'étiage - ce qui semble profiter aux populations d'aloses. Le redressement des captures pourrait en témoigner. L'orientation actuelle des actions qui sont menées en faveur de ces espèces s'articule autour de 3 axes :

- poursuite du programme de rétablissement de la libre circulation des poissons, principalement sur l'axe Adour;

- mise en place d'un plan de gestion des eaux de l'Adour et d'un débit de crise minimum pour assurer des conditions de productions piscicoles « minimales ";

- amélioration des connaissances sur les deux espèces et sur les captures (CPUE) pour disposer de séries chronologiques susceptibles d'indiquer les tendances de l'évolution des stocks.

\section{Lamproie marine}

Seule la lamproie marine, uniquement exploitée au moyen de filets par des pêcheurs professionnels, est répertoriée sur le bassin Adour. Aucune étude spécifique n'a été engagée pour améliorer les connaissances sur la biologie de cette espèce, ni sur la présence éventuelle de la lamproie fluviale. Toutefois, la connaissance des zones de reproduction a fait l'objet d'une cartographie (CSP, à paraître) sur les cours d'eau majeurs du bassin. Les déclarations des captures annuelles des marins pêcheurs font apparaître de très fortes fluctuations ( 0,7 à 16 tonnes/an) sans que l'on puisse se prononcer sur l'évolution réelle des stocks (Figure 8). 


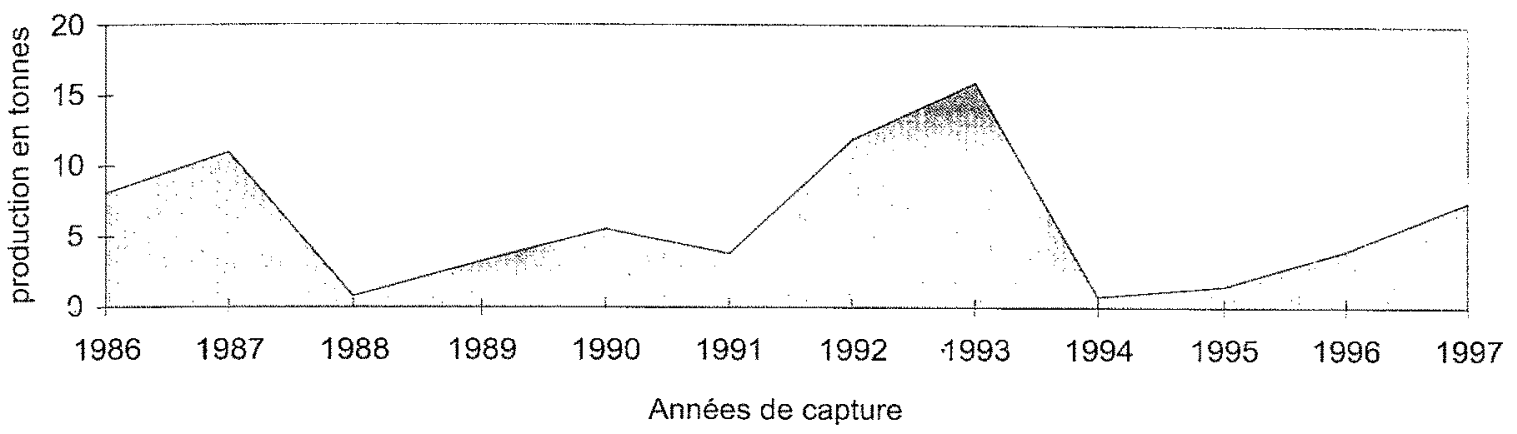

Figure 8

Variations des débarquements de lamproies marines effectués par les marins pêcheurs de l'estuaire de l'Adour de 1986 à 1997 (déclaration).

\section{Figure 8}

\section{Variation of sea lamprey catches by professional fishery in Adour estuary from 1986 to 1997 (declaration).}

Les orientations actuelles concernant ce poisson sont une meilleure évaluation des captures fluviales et des propositions réglementaires visant à préserver les zones de reproduction (arrêté de biotopes).

\section{CONCLUSION}

La mise en place du COGEPOMI Adour réunissant tous les partenaires concernés par les ressources halieutiques constitue une avancée importante vers une gestion rationnelle des stocks d'amphihalins. L'analyse de la situation des migrateurs sur le bassin de l'Adour montre clairement que nous ne maitrisons pas tous les outils techniques et scientifiques pour gérer les stocks de migrateurs amphihalins. Des avancées très significatives ont cependant été réalisées pour le saumon.

Un système performant de récolte d'informations statistiques sur les captures existe sur la partie maritime de l'Adour. Il doit être étendu à la zone fluviale. La mise en place d'un Suivi National de la Pêche aux Engins en zone fluviale centralisé par le C.S.P., dès 1999, est l'occasion de compléter ce dispositif. Un relais local doit cependant être trouvé pour assurer la cohérence et la pertinence des données. Cet outil nouveau devrait permettre, à l'échelle du bassin, d'obtenir des séries chronologiques sur les captures, capables de renseigner les gestionnaires sur les évolutions de stocks.

Les importants investissements menés dans la reconquête de la libre circulation des poissons ont participé au maintien des populations de migrateurs. Ils ont certainement profité à l'ensemble des poissons de nos cours d'eau. Ces efforts doivent être poursuivis et s'accompagner d'actions capables de valoriser les techniques acquises, d'améliorer nos connaissances sur la biologie des poissons et d'évaluer les efforts entrepris.

\section{BIBLIOGRAPHIE}

ANONYME, 1998. Définition d'une cible d'échappement et estimation des contraintes pour la pêche du saumon atlantique sur le bassin versant de l'Adour et de la Nivelle. Rapport CSP/IFREMER/INRA, 22 p. + annexes. 
BARRACOU D., 1999. Stations de contrôle des migrations de poissons - Bassins de l'Adour et de la Nivelle. Rapport MIGRADOUR, $47 \mathrm{p}$.

BOUSQUET B., MARTY A., 1987. Le point sur les connaissances des populations naturelles de saumons dans le bassin de l'Adour. In : THIBAULT M. et BILLARD R. (Eds), La restauration des rivières à saumons, INRA Publ., Paris, 65-76.

CHANSEAU M., 1998. Suivi par radiopistage de la migration anadrome du saumon atlantique sur la partie amont du gave de Pau. Rapport MIGRADOUR, 52 p. + annexes.

CHIMITS P., 1936. Remarques sur le saumon et l'Adour (A suivre). Bull. Fr. Piscic., 99, 57-68.

CHIMITS P., 1936. Remarques sur le saumon et l'Adour (Suite et fin). Bull. Fr. Piscic., 100, 92-98.

DANIEL P., BELLET C., DE CASAMAJOR M.N., SANCHEZ F., PROUZET P., CHARRON M.H., MARTINET J.P., VIGNES J.C., 1998. Infestation parasitaire de l'anguille sur le bassin de l'Adour et sur certains cours d'eau côtiers des Landes et des Pyrénées Atlantiques. Rapport MIGRADOUR, 62 p. + annexes.

DARROLLES V., 1997. Etude de la truite de mer (Salmo trutta L.) sur les bassins des Gaves et des Nives. Rapport de DESS « Dynamique des écosystèmes aquatiques ", UPPA, $78 \mathrm{p}$.

PROUZET P., MARTINET J.-P., DE CASAMAJOR M.-N., POUSSIN L., 1998. Rapport sur la pêche des marins pêcheurs dans l'estuaire de l'Adour en 1997. Rapport IFREMER/St-Pée, $44 \mathrm{p}$.

VIBERT R., 1950. Recherches sur le saumon de l'Adour (Salmo salar L.) (Ages, croissance, cycle génétique, races) 1942-1948. Ann. St. Cent. Hydro. appl., 3, 27-148. 